Europhysics Letters

PREPRINT

\title{
Two dynamic exponents in the resistive transition of fully frustrated Josephson-junction arrays
}

\author{
E. Granato ${ }^{1}$ and D. Domínguez ${ }^{2}$ \\ 1 Laboratório Associado de Sensores e Materiais, Instituto Nacional de Pesquisas Es- \\ paciais - 12201-190 São José dos Campos, São Paulo, Brazil \\ 2 Centro Atômico Bariloche - 8400 San Carlos de Bariloche, Rio Negro, Argentina
}

PACS. 74.81.Fa - Josephson junction arrays and wire networks.

PACS. 64.60. Cn - Order-disorder transformations; statistical mechanics of model systems.

PACS. 74.25.Qt - Vortex lattices, flux pinning, flux creep.

\begin{abstract}
We study the resistive transition in Josephson-junction arrays at $f=1 / 2$ flux quantum per plaquette by dynamical simulations of the resistively-shunted-junction model. The current-voltage scaling and critical dynamics of the phases are found to be well described by the same critical temperature and static exponents as for the chiral (vortex-lattice) transition. Although this behavior is consistent with a single transition scenario, where phase and chiral variables order simultaneously, two different dynamic exponents result for phase coherence and chiral order.
\end{abstract}

Phase transitions in two-dimensional Josephson junction arrays (JJA) has been a subject

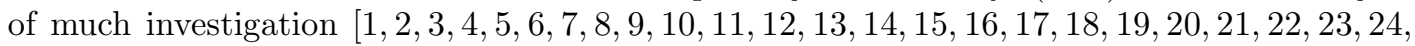
25, 26, 27, 28, 29]. Such arrays can be artificially fabricated [1,2, 3, 4, and are also closely related to superconducting wire networks [5,6. Experimentally, the resistive transition has been the one most extensively studied [2, 3, 4, 5, 6, while theoretically several studies of XY models $[7,8,9,10,11,12,13,14,15,16,17,18,19,20,21,22,23,24,25]$, which describe the JJA, have been done. A significant understanding of these systems has already been achieved by comparing the experiments with the theoretically predicted equilibrium critical behavior with and without an applied magnetic field. However, to a large extent, dynamical critical behavior remains much less explored, particularly in the frustrated case (finite magnetic field). It is well known that while static critical phenomena depend on the spatial dimensionality as well as on the symmetry of the order parameter, the dynamic universality class will depend upon additional properties which do not affect the statics as, for example, conservation laws for the order parameter 30]. Thus, testing the universality hypothesis of dynamical critical behavior requires the study of specific dynamical models. In JJA, the physically relevant dynamical model for the phase dynamics has not been unambiguously identified [26, 27, 16, 17. One would expect that, at least for an array of ideal tunnel junctions, the ResistivelyShunted-Junction (RSJ) model of current flow between superconducting grains would be a more physical representation of the system [28. In experiments, the resistive transition in JJA is usually identified from the behavior of the current-voltage (I-V) characteristics near

(C) EDP Sciences 
the critical temperature. The divergent correlation length determines both the linear and nonlinear resistivity sufficiently close to the transition. To interpret the data and determine the underlying equilibrium transition, the scaling theory of Fisher et al. 31] has been widely used. For JJA at zero magnetic field, the resistive transition is in the Kosterlitz-Thouless (KT) universality class [1,7,28,29. Studies of the critical dynamics, either with Monte Carlo (MC) dynamics [15] or with RSJ dynamics [17, find a behavior consistent with the dynamical theory of the KT transition. The exponent of the current-voltage (I-V) relation, $V \sim I^{a}$, at the transition, assuming the universal value $a=z+1=3$, corresponds to a dynamic exponent $z=2$ in the resistivity scaling theory 31 .

However, in frustrated Josephson-junction arrays (FJJA), corresponding to $f=1 / 2$ flux quantum per plaquette, besides the phase variables, the vortex-lattice induced by the external field introduces and additional Ising-like order parameter, the chirality [7, which measures the direction of local current circulation in the array. The ground state consists of a pinned commensurate vortex-lattice corresponding to an antiferromagnetic arrangement of chiralities and vortex-lattice melting corresponds to the chiral order-disorder transition. As a consequence, two distinct scenarios for the phase transition have been proposed [8, 10, 11, 12, 13] : separated phase-coherence and chiral transitions or a single transition where phase and chirality order simultaneously in a different universality class. Since the resistive transition corresponds to the onset of phase coherence, the later scenario where the critical dynamics should involve coupled variables may lead to important consequences for the resistivity scaling near the transition that can be detected experimentally. Previous numerical studies of the I-V characteristics, obtained either with RSJ dynamics [19 20] or with MC dynamics 21, were performed only for small system sizes $(L \leq 16)$; while other works have studied the shorttime dynamics of chirality [18, and the non-equilibrium transitions at large currents [22]. In particular, the studies with RSJ dynamics used free boundary conditions to impose a driving current. This leads to significant additional dissipation due to boundary effects 32, specially in small system sizes. In this letter, we study in detail the critical dynamics and resistivity scaling in FJJA by numerical simulation of the RSJ dynamics with periodic boundary conditions [23, 24] including large systems sizes $(L=64,128)$. This allowed us to find the following remarkable and unexpected results for the dynamical properties: (i) the current-voltage behavior is well described by a resistive transition corresponding to the chiral transition; (ii) two different dynamic exponents, $z_{X Y} \sim 1$ and $z_{c h} \sim 2$, are found for phase and chiral variables, respectively; and (iii) at the transition, the exponent of the I-V power-law, $V \sim I^{a}$, is $a=z_{X Y}+1 \approx 2$ rather than $a=3$ as for the unfrustrated case. We discuss an interpretation of these results within the single transition scenario including a multicritical point and also suggest possible explanations within the double transition picture.

We consider a square two-dimensional array described by the overdamped RSJ model with current conservation at each node [28. The equations of motion for the phases $\theta_{i}$ of the superconducting order parameter located at node $i$ of the square lattice can be written as

$$
\frac{\hbar}{2 e R_{o}} \sum_{j}\left(\dot{\theta}_{i}-\dot{\theta}_{j}\right)=-\sum_{j}\left[I_{o} \sin \left(\theta_{i}-\theta_{j}-A_{i j}\right)+\eta_{i j}\right]
$$

where $R_{o}$ is a uniform shunt resistance, $\eta_{i j}(t)$ is a thermal noise with correlations $\left\langle\eta_{i j}(t) \eta_{k l}\left(t^{\prime}\right)\right\rangle=$ $2 k_{B} T / R_{o} \delta_{i j, k l} \delta\left(t-t^{\prime}\right), I_{o}$ is the junction critical current, $A_{i j}=\int_{i}^{j} \mathbf{A} . d \mathbf{l}$ is the line integral of the vector potential, and $\sum_{i j} A_{i j}=2 \pi f$, with the sum taken around each elementary plaquette of the lattice. Dimensionless quantities are used with time in units of $\tau=\hbar / 2 e R_{o} J_{o}$, current in units of $I_{o}$, voltages in units of $R_{o} I_{o}$ and temperature in units of $\hbar I_{o} / 2 e k_{B}$. A total current $I$ is imposed uniformly in the array using fluctuating periodic boundary condi- 

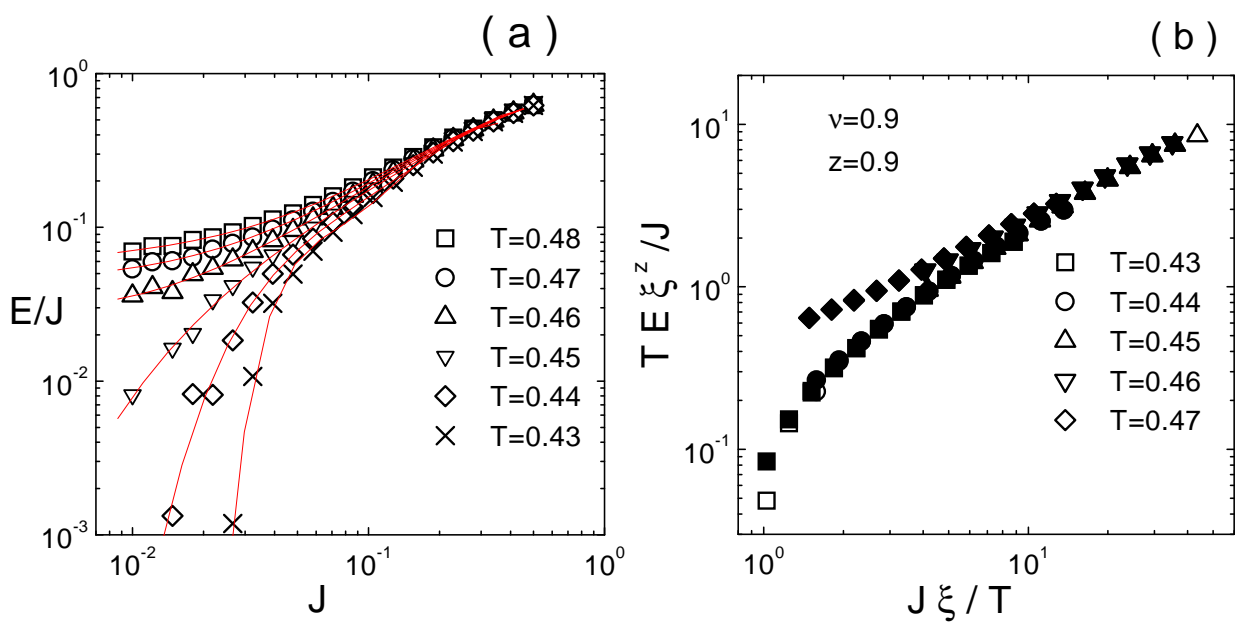

Fig. 1 - (a) Nonlinear resistivity $E / J$ as a function of temperature for system size $L=64$. (b) Scaling plot of the data for the smallest current densities. Open symbols correspond to $L=64$ and filled ones to $L=128$.

tions [23 24] with current density $J=I / L$, where $L$ is the system size and the average electric field $E$ is obtained from the voltage $V$ across the system as $E=V / L=(\hbar / 2 e)\langle d \Theta / d t\rangle$, with $\Theta$ the global phase difference or twist 23 . We integrate the dynamical equations with a second order Runge-Kutta-Helfand-Greenside method with time step $\Delta t=0.07 \tau$, taking $10^{6}$ steps for the lowest currents while for large currents $2 \times 10^{4}$ steps were enough for proper equilibration. The results were averaged over $5-10$ different initial configurations of the phases and sizes of $L=8$ to $L=128$ were considered.

Fig. 1(a) shows the temperature dependence of the nonlinear resistivity $E / J$ near the chiral transition temperature, estimated previously from equilibrium Monte Carlo simulation [8], $T_{c h}=0.455$. Qualitatively, the linear resistance $R_{L}=\lim _{J \rightarrow 0} E / J$, tends to a finite value at high temperatures but extrapolates to very low values at lower temperatures, independent of system size, consistent with the existence of a resistive transition in the range $T_{c}=0.44$ to 0.46. In the double transition scenario, where the phase-coherence transition is expected to be in the KT universality class, the estimate of the KT critical temperature $T_{K T}=0.446$ from Monte Carlo simulations 13, is very close to $T_{c h}$ and so without further analysis the true resistive critical temperature, at $J=0$ current drive, could be consistent with both estimates. However, we note that the IV curve for $T_{c h}>T=0.45>T_{K T}$ tends to zero resistivity for $J \rightarrow 0$, while the IV curve for $T=0.46>T_{c h}$ tends to finite resistivity for $J \rightarrow 0$. This already suggests that the resistivity transition occurs at $T_{c h}$ rather than at $T_{K T}$. In any case, the asymptotic critical behavior can be inferred more adequately from a scaling analysis of the nonlinear resistivity. According to the scaling theory [31, measurable quantities scale with the diverging correlation length $\xi$ and the relaxation time $\tau \propto \xi^{z}$, near the transition temperature, where $z$ is the dynamical critical exponent. Then, the nonlinear resistivity should satisfy the scaling form

$$
T \frac{E}{J}=\xi^{-z} g_{ \pm}\left(\frac{J}{T} \xi\right)
$$

in two dimensions, where the + and - correspond to the behavior above and below the transition, respectively. For a transition in the KT universality class, the correlation length 
should diverge exponentially as $\xi \propto \exp \left(b /\left|T / T_{c}-1\right|^{1 / 2}\right)$, while otherwise a power-law behavior is expected $\xi \propto\left|T / T_{c}-1\right|^{-\nu}$, with an exponent $\nu$ to be determined. Thus, a scaling plot according to Eq. (2) can be used to verify the dynamic scaling hypotheses and the assumption of an underlying equilibrium transition. Such scaling plot is shown in Fig. 1(b), in the temperature range closest to $T_{c h}$ and smallest current densities, assuming the correlation length $\xi$ has a power-law divergence with $T_{c}=T_{c h}$ and using $\nu$ and $z$ as adjustable parameters so that the best data collapse is obtained. Similar scaling analysis assuming a KT correlation length and fixing $T_{c}$ at the estimates of $T_{c h}$ or $T_{K T}$ do not result in a good data collapse 33. From this scaling analysis, we estimate $\nu=0.9(1)$ and the dynamical critical exponent $z=0.9(2)$. We note that the static exponent $\nu$ is consistent with estimates of the chiral transition from equilibrium Monte Carlo simulations [8. However, in simulations with MC dynamics 21 a dynamic exponent $z \sim 2$ was found, but this may correspond to a different dynamics and also note that small systems with $L=8-14$ were analyzed. As it is well known, finite-size effects are very important sufficiently close to the transition when the correlation length $\xi$ reaches the system size $L$. Previous simulations with RSJ dynamics [19 20] considered very small sizes $(L=6-16)$. In our case, as shown in the Fig. 1(b), the two largest system sizes $L=64$ and $L=128$ give the same data collapse and so finite size effects are not dominant for this range of temperatures and current densities in our data. Although the above scaling analysis for large system sizes already suggests that the resistive transition temperature $T_{c}$ is very close to $T_{c h}$ with $z<2$, in absence of a completely satisfactorily determination of $T_{c}$ from static critical behavior [10 11], 13, from now on, we will assume 34 $T_{c}=T_{c h}$ and explore to which extent this give us consistent results from dynamics, including finite-size effects. At $T_{c}$, the correlation length $\xi$ will be cut off by the system size in any finite system. From Eq. (2), the nonlinear resistivity at $T_{c}$ should then satisfy the scaling form

$$
T \frac{E}{J}=L^{-z} g\left(\frac{J}{T} L\right)
$$

We have tested the scaling form of Eq.(13) at $T_{c}=T_{c h}$ for different sizes $L=8-128$ and we found a very good finite size scaling with the same dynamic exponent $z=0.9(1)$ as shown in Fig. 2(a). On the contrary, when the I-V curves were calculated at $T=T_{K T}$ [33, it was not possible to obtain a reasonable data collapse using Eq.(3) for $z$ values in the range $[0.5,4]$.

It could still be argued that our estimate of $z$ is based on a scaling analysis of the nonlinear $\mathrm{I}-\mathrm{V}$ characteristics, which is a nonequilibrium property, and so does not reflect the underlying equilibrium transition. However, additional equilibrium calculations of the linear resistance at $T_{c h}$ give a consistent estimate. From Eq. (3), the linear resistance $R_{L}=\lim _{J \rightarrow 0} E / J$ at $T_{c}$ should scale as $R_{L} \propto L^{-z}$. The linear resistance can be obtained from the Kubo formula of equilibrium voltage fluctuations as

$$
R_{L}=(1 / 2 T) \int d t\langle V(t) V(0)\rangle,
$$

without an imposing driving current, and can also be conveniently determined from the longtime fluctuations of the phase difference across the system [25. Fig. 2(b), shows the finite size behavior of $R_{L}$. A power-law fit gives $z=0.89(6)$ which is in fact consistent with the estimate from the I-V scaling and suggests therefore that the value of $z$ corresponds to the underlying equilibrium dynamical behavior. For comparison, it is also shown in Fig. 2(b) the behavior for the unfrustrated case, $f=0$. In this case the resistive transition is in KT universality class and a dynamical exponent $z=2$ is expected, independent of the particular dynamics [15 17]. Indeed, for $f=0$, the same power-law fit at the critical temperature $T_{c}=0.887$ estimated previously from Monte Carlo simulations [35] gives $z=2.0(1)$. 
( a )

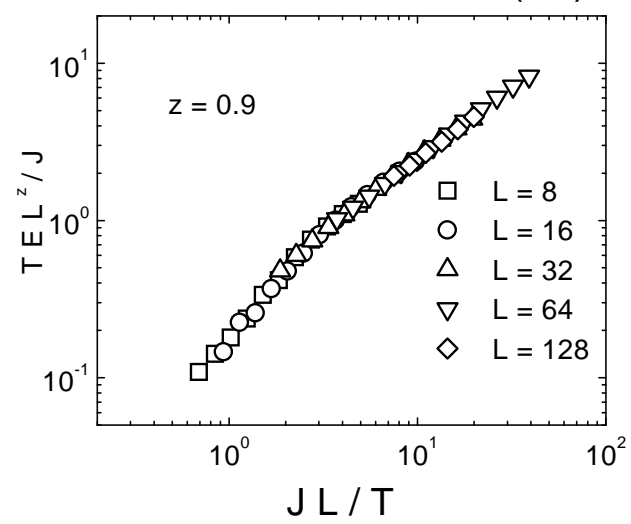

( b )

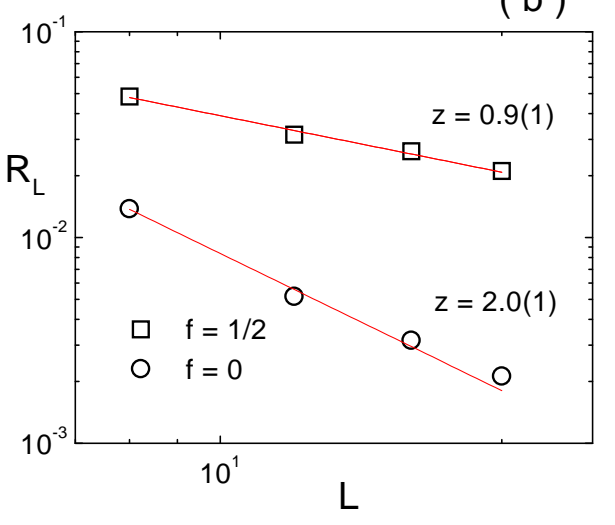

Fig. $2-$ (a) Scaling plot of the nonlinear resistivity $E / J$ at $T_{c}=T_{c h}=0.455$ for different system sizes $L$. (b) Linear resistance as a function of system size at the critical temperatures $T_{c}=T_{c h}$ for $f=1 / 2$ and $T_{c}=0.887$ for $f=0$. Power-law fits give estimates exponent $z$.

Finally, to further verify that the estimate of $z$ does in fact reflect critical phase fluctuations near the transition, we have also performed equilibrium calculations of the phase autocorrelation function $C_{X Y}(t)=\langle\vec{S}(0) \cdot \vec{S}(t)\rangle$, where $\vec{S}=\sum_{i} \vec{s}_{i}$ and $\vec{s}=(\cos (\theta), \sin (\theta))$. The relaxation time $\tau_{X Y}$ can be obtained from the exponential decay $C_{X Y}(t) \propto \exp \left(-t / \tau_{X Y}\right)$ at long times. Similar calculations were also performed for the chirality autocorrelation function $C_{c h}(t)$ to obtain $\tau_{c h}$, with the local chirality defined as $\chi=\sum_{<i j>}\left(\theta_{i}-\theta_{j}-A_{i j}\right) / 2 \pi$, where the summation is taken over the elementary plaquette of the lattice and the gauge-invariant phase difference is restricted to the interval $[-\pi, \pi]$. From dynamic finite-size scaling, the relaxation time should scale at $T_{c}$ as $\tau \propto L^{z}$, from which the $z$ can be estimated from the slope in a $\log \log$ plot. Fig. 3 shows the finite-size behavior of the relaxation time at $T_{c h}$ for the phases and chiralities. From a power-law fit we obtain $z_{X Y}=1.1(1)$ from the phase relaxation time $\tau_{X Y}$ which is indeed consistent with the estimate of $z$ from the resistivity scaling discussed above. Naively, if the two transitions happen at the same temperature, one would expect that the same dynamic exponent should also hold for the chiral relaxation time. Surprisingly, however, the estimate from the chiral relaxation time in Fig. 3 is quite different, $z_{c h}=2.1(1)$.

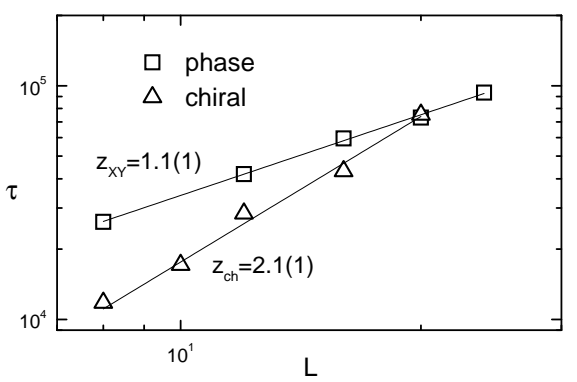

Fig. 3 - Finite-size behavior of the phase and chiral relaxation times, $\tau_{X Y}$ and $\tau_{c h}$ respectively, at the critical temperature $T_{c}=T_{c h}$. Power-law fits give estimates of the dynamical exponents $z_{X Y}$ and $z_{c h}$. 
Nevertheless, this value is consistent with the result of short-time dynamics obtained from MC simulations [18.

Thus, our numerical results imply a single transition at $f=1 / 2$ with two dynamic exponents, $z_{X Y} \sim 1$ and $z_{c h} \sim 2$. It should be noted that two dynamic exponents at the transition does not necessarily imply a breakdown of dynamic scaling in the restricted sense [30, since only the chirality is an order parameter which develops true long-range order below the transition. In two-dimensions, the phases can only develop quasi-long-range order. Since $z_{c h}>z_{X Y}$, the longest relaxation time is still determined by the order parameter. However, extended dynamic scaling 30] still applies to the resistivity and a different dynamic exponent is possible. Whether this only holds for the dynamics of RSJ model studied here or also for other dynamical models will require further work. However, we should mention that different dynamic exponents for coupled order parameters have already been found previously at multicritical points in magnetic systems [37. This would suggest that a possible explanation for two dynamic exponents at the transition of the FJJA may rely on the existence of a multicritical point in the phase diagram of the relevant effective model describing the transition. Interestingly enough, the coupled XY-Ising model [8] which is expected to describe the static critical behavior of the FJJA, does have a multicritical point and could be a useful framework for future investigations of the dynamical universality class of FJJA.

Although the single transition scenario provides a consistent interpretation of our data, we should emphasize that the alternative separated transitions scenario can not be ruled out. In fact, recent work by Korshunov [14] argues for this scenario on good theoretical grounds. If this picture turns out to be the correct one then, we believe, there are two possible explanations for our findings: 1) the KT transition is actually much closer to $T_{c h}$ than estimated previously and so the transitions can not be resolved within the accuracy of our data; 2) the scaling theory of 31 is not valid for the present case and should therefore be enlarged to include the interplay of two divergent length scales at nearby temperatures 13, 14 which can lead to crossover effects at small length and time scales. However, it remains unclear how the resistivity scaling can be so well described by the chiral transition temperature including data for different temperature and systems sizes.

In conclusion, we find that the resistivity scaling and critical dynamics of FJJA are well described by the critical temperature corresponding to the chiral (vortex-lattice) transition. Two dynamic exponents, $z_{X Y} \sim 1$ and $z_{c h} \sim 2$, are found for phase-coherence and chiral order, respectively, and, at the transition, the exponent of the I-V power-law, $V \sim I^{a}$, is $a=z_{X Y}+1 \approx 2$ rather than $a=3$ as for the unfrustrated case. One implication of these results for transport experiments is that the usual method of locating the critical temperature from the value corresponding to a nonlinear I-V exponent $a=3$, will lead to a significant underestimate [36]. It is worth mentioning that some experiments in overdamped arrays 3 ] find a value of $a \approx 2$ at $T_{c}$ in agreement with our results. In addition, resistivity scaling of experimental data on wire networks 6 is also consistent with power-law correlation as found here but with $z \sim 2$. Since the dynamics of wire networks are different than the RSJ dynamics, this value of $z$ may be the result of a different dynamical universality class. Further detailed I-V measurements combined with magnetic properties, which could in principle probe the chiral transition, are needed to test our results.

$$
* * *
$$

The work of E.G. was supported by FAPESP and of D.D. by CONICET and ANPCyT (PICT99 03-06343). 
E. Granato and D. Domínguez: Dynamic exponents in frustrated Josephson arrays7

\section{REFERENCES}

[1] Newrock R. S . et al., Solid State Physics, 54 (2000) 263.

[2] van Wees B. J. et al., Phys. Rev. B, 35 (1987) 7291; van Der Zant H. S. J. et al., J. Low Temp. Phys., 82 (1991) 67.

[3] Carini J. P., Phys. Rev. B, 38 (1988) 63; Brown R. K. and Garland J. C., Phys. Rev. B, 33 (1986) 7827.

[4] Lerch Ph. et al., Phys. Rev. B, 41 (1990) 11579.

[5] Yu F. et al., Phys. Rev. Lett., 68 (1992) 2535.

[6] Ling X. S. et al., Phys. Rev. Lett., 76 (1996) 2989.

[7] Teitel S. and Jayaprakash C., Phys. Rev. Lett., 51 (1983) 1999; Phys. Rev. B, 27 (1983) 598.

[8] Granato E. et al., Phys. Rev. Lett. , 66 (1991) 1090; Lee J. et al., Phys. Rev. B, 44 (1991) 4819.

[9] Lee J. et al., Phys. Rev. B, 43 (1991) 11531.

[10] Ramirez-Santiago G. and José J. V., Phys. Rev. Lett., 68 (1992) 1224; 77 (1996) 4849.

[11] Granato E. et al., Physica B, 222 (1996) 266 and references therein.

[12] Lee S. and LeE K.C., Phys. Rev. B, 49 (1994) 15184.

[13] Olsson P., Phys. Rev. Lett., 75 (1995) 2758; 77 (1996) 4850.

[14] Korshunov, S.E., Phys. Rev. Lett., 88 (2002) 167007.

[15] Weber H. et al., Phys. Rev. B, 53 (1996) 8566.

[16] Tiesinga P. H. E. et al., Phys. Rev. Lett., 78 (1997) 519.

[17] Jensen L. M. et al., Phys. Rev. B, 61 (2000) 15412.

[18] Luo H. J. et al., Phys. Rev. Lett., 81 (1998) 180.

[19] Mon K. K. and Teitel S., Phys. Rev. Lett., 62 (1989) 673.

[20] Simkin M. V., Phys. Rev. B, 57 (1998) 7899.

[21] Lee J.R. and Teitel S., Phys. Rev. B, 50 (1994) 3149.

[22] Marconi V. I. and Domínguez D., Phys. Rev. Lett., 87 (2001) 017004.

[23] Domínguez D., Phys. Rev. Lett., 82 (1999) 181; Kıм B. J. et al., Phys. Rev. B, 59 (1999) 11506; Choi, M.Y. et al., Phys. Rev. B, 62 (2000) 5357.

[24] Granato E. and Domínguez D., Phys. Rev. B, 63 (094507) 2001.

[25] Granato E., Phys. Rev. B, 58 (1998) 11161.

[26] Theron R. et al., Phys. Rev. Lett., 71 (1993) 1246.

[27] Beck H., Phys. Rev. B, 49 (1994) 6153.

[28] Shenoy S.R., J. Phys. C, 18 (1985) 5163.

[29] Minnhagen P., Rev. Mod. Phys., 59 (1987) 1001.

[30] Hohenberg P.C. and Halperin B.I., Rev. Mod. Phys., 49 (1977) 435.

[31] Fisher D.S. et al., Phys. Rev. B, 43 (1991) 130.

[32] Simkim M.V. and Kosterlitz J.M., Phys. Rev. B, 55 (1997) 11646; Granato E. et al., Phys. Rev. B, 57 (1998) 3602.

[33] Granato E. and Domínguez D., unpublished.

[34] In general, the most relliable way of determining the dynamic exponent $z$ is to use the known value of $T_{c}$. This is so even for models where the static critical behavior is known exactly as for the Ising model (Lacasse, M.D. et al., Phys. Rev. B 47, 5646 (1993)). For the FJJA considered here, there is no exact solution, but the two competing scenarios [8 9 10 11 [13] imply that a resistive transition occurs either at $T_{c}=T_{c h}$ or $T_{c}=T_{K T}$. Our numerical data does not lead to consistent scaling behavior under the assumption $T_{c}=T_{K T}$ with the current estimates of $T_{K T} 33$.

[35] Weber H. and Minnhagen P., Phys. Rev. B, 37 (1988) 5986.

[36] The experiments in underdamped arrays of [2] were fitted using the KT theory and obtained $a>3$. This leads in their case to an estimate of the critical temperature of $T_{c} \approx 0.3$, which is significantly lower than the theoretical $T_{c} \approx 0.45$.

[37] Huber D.L. and Raghavan R., Phys. Rev. B, 14 (1976) 4068. 